\title{
Clinical Evaluation of a Dentifrice Containing Nhap for the Reduction of Dentin Hypersensitivity
}

\section{Kondyurova Evgenia $V^{1}$, Lisevtsova Julia $V^{1}$ Eliseykina Elena $V^{1}$, Vilikotskiy Anatoliy $E^{2^{*}}$ and Zakirova Svetlana $A^{2}$}

${ }^{1} N$ P Ogarev's Mordovia State University, Russia

${ }^{2}$ SPLAT GLOBAL, LLC, Lotte Plaza, Novinskiy Boulevard, Russia

*Corresponding author: Vilikotskiy Anatoliy E, SPLAT GLOBAL» LLC, Novinskiy Boulevard, 8, 121099 Moscow, Russia, Tel: 8-495-725-66-38

\begin{abstract}
Objective: Compare the cleaning efficiency of toothpaste with hydroxyapatite to evaluate efficacy and safety of two toothpaste (Sensitive Ultra) and (Sensitive White) during tooth brushing, containing $0.1 \%$ and $0.5 \%$ nHAP for the treatment of dentin hypersensitivity.

Methods: Open single-center comparative randomized clinical study in two parallel groups. 60 volunteers at the age of 18 to 65 years participated in the research. This was a 4-week, single-centre, open-label, two-treatment, parallel-group, exploratory study in healthy adult volunteers with self-reported and clinically diagnosed Dentin hypersensitivity. Evaporative, chemical and thermal stimuli were used to measure pain. Measurements were obtained at baseline, 2 weeks and 4 weeks. The assessment of efficacy was measured by Green-Vermillion and Silness-Loe Index, safety was tested by frequency and severity of side effects. The assessment of efficacy was measured by Schiff Index.

Results: The dentifrices showed a statistically significant decrease in sensitivity after 4 weeks $(P<0.05)$. The percent reduction in sensitivity at 4 weeks for the test group was $69.6 \%$ for Sensitive White and $66.7 \%$ for Sensitive Ultra. These results demonstrate that the nHAP-containing dentifrices were effective at reducing sensitivity.
\end{abstract}

\section{Keywords}

Clinical trials, Hydroxyapatite, Hypersensitivity, Dentifrices

\section{Introduction}

Dentin hypersensitivity (DH) has been defined as a "short, sharp pain arising from exposed dentin in response to stimuli, typically thermal, evapora- tive, tactile, osmotic or chemical, and that cannot be ascribed to any other form of dental defect or pathology" [1]. Dentine hypersensitivity is a significant burden for patients and practitioners. Knowing its prevalence and methods for accurate diagnosis is imperative to guide treatment decisions and develop new treatments. A wide range of estimates of prevalence of dentine hypersensitivity was observed in 56 studies, from as low as $1.8 \%$ to as high as $84 \%$, and this heterogeneity could not be completely explained by study characteristics. The meta-analysis revealed that the average prevalence of all studies was 33\% and the best estimate was approximately $10 \%[2,3]$. One of the most frequent patient complaints is related to cold stimuli, although pain may also occur when consuming acidic foods (mainly fruit), sweets and salty foods. Tactile stimulus provocation frequently occurs when patients brush their teeth or rub the sensitive area with a finger nail [4]. DH may disturb patients during eating, drinking and brushing. The prevalence of $\mathrm{DH}$ has been reported to be in the range of 8 to 57 . Hypersensitivity may occur wherever the dentin is exposed by attrition or abrasion, or the root surface is exposed by periodontal disease. In regard to the pain pathogenic mechanism, several theories have been proposed. Nowadays, the most accepted one is Brannstrom's hydrodynamic theory [5]. According to this theory, DH is caused by shifts in the fluid located inside the open dentin tubules. This

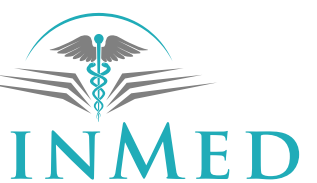

INTERNATIONAL LIBRARY

Citation: Kondyurova EV, Lisevtsova JV, Eliseykina EV, Vilikotskiy AE, Zakirova SA (2019) Clinical Evaluation of a Dentifrice Containing Nhap for the Reduction of Dentin Hypersensitivity. Int J Oral Dent Health 5:104. doi.org/10.23937/2469-5734/1510104

Accepted: December 02, 2019: Published: December 04, 2019

Copyright: (C) 2019 Kondyurova EV, et al. This is an open-access article distributed under the terms of the Creative Commons Attribution License, which permits unrestricted use, distribution, and reproduction in any medium, provided the original author and source are credited. 
movement would trigger pulp nerve fibres, causing pain symptoms to the patient.

Although there are many therapeutic options for the treatment of $\mathrm{DH}$, the management of this condition is still a problematic issue. This is due not only to the fact that its etiology has not been yet completely understood, as the actual 'mode of action' of the therapeutic agents remains unclear, but also to the scarce, ambiguous and not very conclusive scientific evidence available concerning the clinical efficacy of desensitizing products. Even though there have been proposed several categories for the management of the $\mathrm{DH}$, currently, there are no evidence-based therapeutic protocols involving $\mathrm{DH}$ patients that allow us to achieve predictable results [6].

Toothpastes and mouth rinses are available for home use. Their mechanisms of action fall under two basic categories, being nerve desensitization and occlusion of exposed dental tubules. To allow fluid movement that sensitizes the nerves, the tubules must be patent. Thus products for home use can relieve sensitivity by occluding the exposed dentinal tubules through mechanical formation of a natural smear layer by burnishing dentin over the tubule openings and forming insoluble materials that precipitate in the tubules and on the surface. One of these compounds is nanohydroxyapatite (nHAP). nHAP acts as a calcium and phosphate reservoir, helping to maintain a topical state of supersaturation of these ions with respect to tooth minerals, and thus causing deposition on the surface. It can function by directly filling up micropores in dentin, and inside the pores it acts as a template in calcium phosphate formation by continuously attracting large amounts of calcium and phosphate ions from the saliva and other mineralizing solutions to the tooth tissue, thus promoting crystal integrity and growth [7].

A number of clinical studies have been conducted on the efficacy of different dentifrices for the relief of hypersensitivity. This study was designed to investigate the effects of two nHAP-containing dentifrices in patients with moderate levels of dentin hypersensitivity.

\section{Materials and Methods}

\section{Study design}

This was 4-week, single-centre, open-label, two-treatment, parallel-group, exploratory study in healthy adult volunteers with self-reported and clinically diagnosed $\mathrm{DH}$. The study was conducted at the Ogarev Mordovia State University, Saransk, Russia. It was approved by the university's independent ethics committee before initiation and conducted in accordance with the Declaration of Helsinki.

SPLAT Professional Sensitive Ultra (Dentifrice A) contained $0.5 \% \mathrm{nHAP}$ and Splat Professional Sensitive White (Dentifrice B) containing 0.1\% nHAP were man-
Table 1: Demographic data for subjects completing study.

\begin{tabular}{|l|l|l|l|l|}
\hline \multirow{2}{*}{ Group } & \multicolumn{3}{|l|}{ Number of subjects } & Age \\
\cline { 2 - 5 } & Men & Women & Mean & Range \\
\hline Dentifrice A & 18 & 12 & 39.3 & $18-65$ \\
\cline { 2 - 5 } & $(60 \%)$ & $(40 \%)$ & & \\
\hline Dentifrice B & 16 & 14 & 39.6 & $18-63$ \\
\cline { 2 - 5 } & $(53.3 \%)$ & $(46.7 \%)$ & & \\
\hline
\end{tabular}

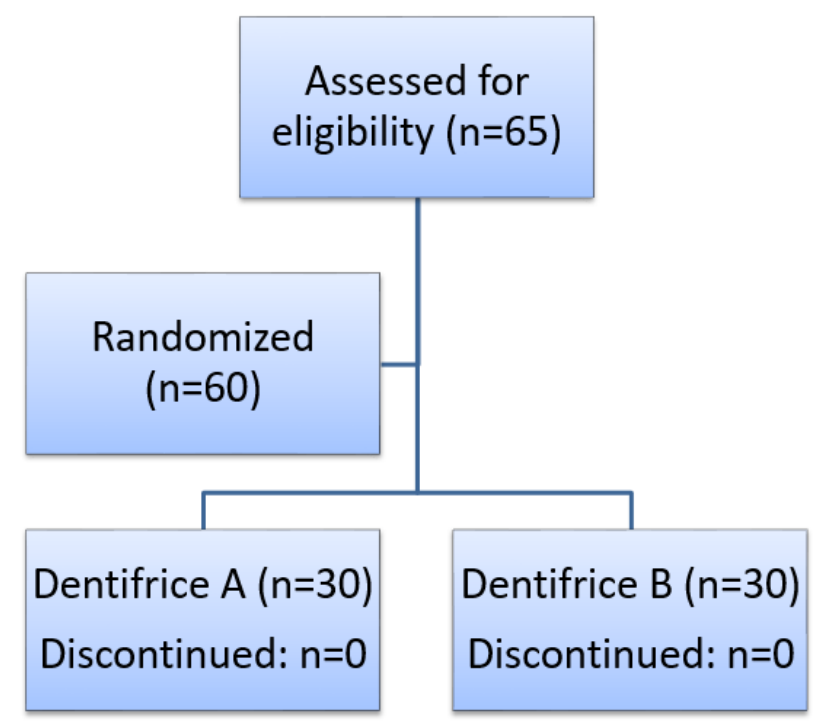

Figure 1: Participant flow through the study.

ufactured by Organic Pharmaceuticals (Novgorod region, Russia) as well as SPLAT Professional Complete medium hardness toothbrushes (further $\mathrm{x} \% \mathrm{nHAP}$ ). Test dentifrices were supplied to study volunteers in their original commercial packaging with a study-specific label detailing protocol number, product code, storage conditions, and precautionary information including an emergency contact telephone number.

At screening visit baseline demographic information was collected: Sex, age, concomitant therapy. 60 volunteers participated in the research-30 in a group obtained Dentifrice A and 30 Dentifrice B (Table 1). Participant flow through the study is shown in Figure 1. Eligible subjects were required to attend the study site on three occasions: Screening/baseline, after first use dentifrice, 2 and 4 weeks after the baseline visit. At the screening visit, each subject provided written informed consent to participate in the study. Demographic characteristics, medical history and use of concomitant medications were recorded and an oral examination was completed. To determine eligibility, each subject's dentition was evaluated sequentially for evidence of erosion, abrasion or facial/cervical gingival recession; gingival health; tooth mobility; and sensitivity to an air-blast stimulus (as indicated by a 'yes' response when the subject was questioned about discomfort following stimulation. Subjects with at least two eligible sensitive teeth were supplied 
with a toothpaste (A or B) and a soft nylon toothbrush to use twice daily (morning and evening) for a period of 4 weeks.

Before the first application the following indices and measurements were performed: OHI-S (Green-Vermillion Index), Silness-Loe Index, CPITN (Community Periodontal Index of Treatment Needs), Shtorina Index.

The enamel remineralization effect was carried out by means of test of enamel resistance (TER). The vestibular surface of one of the upper central incisors was cleaned of plaque using a cotton ball moistened with hydrogen peroxide solution, and then the tooth was dried with a cotton ball. The tooth was isolated from the saliva with cotton rolls. A drop of $1 \% \mathrm{HCl}$ solution with a diameter of $1.5-2 \mathrm{~mm}$ was applied to the center of the vestibular tooth surface with a micropipette. After 5 seconds, the drop was washed with distilled water. The tooth surface was dried with a cotton ball. On the area of enamel, which was treated, 1 drop of $1 \%$ solution of methylene blue was put. The dye was immediately removed with a dry cotton ball in one motion, tightly pressing the ball to the surface of the tooth.

In daylight, the color of the treated area was compared to the shades of a standard 10-point scale. Estimated acid resistance of enamel in points from 1 to 10 (from pale blue to dark blue). The procedure was carried out on visits 1 and 3 .

According to the result, the patient was referred to one of 4 dispensary groups:

1. 1-3 points - high acid resistance of enamel;

2. 4-5 points - moderate acid resistance of enamel;

3. 6-7 points - low acid resistance of enamel;

4. More than 7 points - very low acid resistance of enamel.

At the baseline visit, subjects were assessed for continuing eligibility. Shtorina Index is based on Schiff sensitivity index evaluating all teeth at least for reaction to temperature stimulus. It was calculated as a ratio between the sum of all teeth Schiff scores to all teeth evaluated, thus it could value from 0 to 3 continuously in consideration of the fact that not all teeth could have hyperaesthesia.

Sensitivity to mechanical, chemical and thermal stimuli was assessed using the 4-point index for each tooth, scored as: 0 = subject does not respond to temperature, chemical, mechanical stimulus; 1 = subject responds to temperature stimulus; 2 = subject responds to temperature stimulus and chemical stimulus; 3 = subject responds to temperature stimulus, chemical stimulus and mechanical stimulus [8,9].

Following an examination, the sensitivity of all teeth was evaluated by the subject's response to a tactile stimulus [10], to an evaporative (air) stimulus (assessed by examiner-based Schiff sensitivity score [11]) and chemical stimulus [12]. There was a minimum of $5 \mathrm{~min}$ delay between the end of the tactile assessment and the start of the evaporative (air) assessment to allow the tooth to recover.

Randomization table was prepared using IBM SPSS 22 software for Windows. Clinical sites received sealed envelopes with randomization number on it. Inside the envelopes were put inserts with information on distribution of the volunteer to one or another group.

\section{Statistics}

The statistical analysis was performed using IBM SPSS 22 for Windows. The data were analyzed using Mann-Whitney $U$ test with $5 \%$ alpha-level. The level of significance was set at 0.05 .

Sample size calculation was not performed as the study was conducted with new product and there had not been similar studies to use in the calculation. The power of study was determined at the planning stage and is equal to 0.9 .

\section{Safety}

Spontaneously reported adverse events (AEs) and any abnormalities in the OST examination were recorded from the first supervised brushing with the toothpaste at the screening visit until 5 days after the last administration of study product. The investigator assessed the relationship between investigational product and the occurrence of each $A E$ using clinical judgment, and graded the intensity of the AE as mild, moderate or severe.

During the study, subjects were not permitted to use any oral care products other than those provided or any dental products (including home remedies) intended for treating sensitive teeth; they were only permitted to use dental floss for the removal of impacted food. Subjects were requested not to have any non-emergency dental treatment (including prophylaxis) during the course of the study. Subjects were asked to refrain from excessive alcohol consumption for $24 \mathrm{~h}$ before each scheduled assessment visit; from all oral hygiene procedures and from chewing gum for at least $8 \mathrm{~h}$; and from eating, drinking and smoking for at least $2 \mathrm{~h}$.

Eligible subjects were 18-65 years-old, in good general health with no clinically significant and relevant abnormalities of medical history or oral examination. They were required to have a self-reported history of $\mathrm{DH}$ lasting more than 6 months but not more than 10 years.

General exclusion criteria included: Pregnancy or breastfeeding; any known or suspected intolerance or hypersensitivity to the study materials or ingredients; participation in another clinical study or receipt of an 
investigational drug within 30 days, or participation in a desensitizing-treatment study within 8 weeks of the screening visit; use of a sensitivity toothpaste within 8 weeks of screening; presence of chronic debilitating disease that could have affected study outcomes; any condition or daily use of medication that was causing xerostomia and daily use of any medication or traditional herbal ingredients or treatments that could have interfered with the perception of pain. General oral exclusions included: Dental prophylaxis within 4 weeks of screening; presence of dental implants; tongue or lip piercings; gross periodontal disease or treatment of periodontal disease (including surgery) within the past 12 months; scaling or root planning within 3 months of screening and teeth bleaching within 8 weeks of screening. Specific dentition exclusions at screening included: Teeth with current or recent caries, or reported treatment of decay in the past 12 months; teeth with exposed dentine but with deep, defective or facial restorations; teeth used as abutments for fixed or removable partial dentures; teeth with full crowns or veneers, or- thodontic bands or cracked enamel and sensitive teeth with contributing etiologies.

\section{Results}

As it could be seen from Table 1 demographic characteristics were comparable between groups as well as concomitant therapy-volunteers did not have acute or chronic in acute phase diseases, therefore there was no need in any specific concomitant therapy during the study.

Each group contained participants with teeth hypersensitivity of various severities.

Table 2 shows the scores at each examination period for 60 patients for toothpaste Sensitive White (Dentifrice A). Sensitivity scores declined steadily after the use of dentifrices was begun, decreasing by almost $70 \%$ in the end of the study period. After 4 weeks of treatment the $\mathrm{OHI}$ score shown a $17.0 \%$ reduction while other parameters have not changed significantly (Figure 2).

Table 2: Sensitive white.

\begin{tabular}{|l|l|l|l|l|}
\hline & Baseline & After first use dentifrice & Visit 2 & Visit 3 \\
\hline $\mathrm{OHI}^{\mathrm{a}}$ & $1.04 \pm 0.33$ & $0.91 \pm 0.25$ & $0.89 \pm 0.23^{*}$ & $0.88 \pm 0.28^{*}$ \\
\hline Sensitivity & $0.70 \pm 0.60$ & - & $0.63 \pm 0.61$ & $0.23 \pm 0.50^{*}$ \\
\hline Enamel mineralization & $3.10 \pm 1.58$ & - & - & $3.07 \pm 1.51$ \\
\hline CPITN $^{b}$ & $1.07 \pm 1.05$ & - & $1.00 \pm 1.02$ & $1.07 \pm 1.11$ \\
\hline
\end{tabular}

${ }^{\mathrm{a}} \mathrm{OHI}=$ Oral Hygiene Index; ${ }^{\mathrm{b}} \mathrm{CPITN}=$ Community Periodontal Index of Treatment Needs; ${ }^{*} \mathrm{p}<0.05$ compared to baseline.

Table 3: Sensitive ultra.

\begin{tabular}{|l|l|l|l|l|}
\hline & Baseline & After first use dentifrice & Visit 2 & Visit 3 \\
\hline $\mathrm{OH}^{\mathrm{a}}$ & $1.06 \pm 0.38$ & $0.94 \pm 0.30$ & $0.95 \pm 0.34$ & $0.88 \pm 0.29^{*}$ \\
\hline Sensitivity & $0.77 \pm 0.57$ & - & $0.53 \pm 0.51^{*}$ & $0.23 \pm 0.43^{*}$ \\
\hline Enamel mineralization & $2.83 \pm 1.49$ & - & - & $2.4 \pm 1.13$ \\
\hline CPITN $^{\mathrm{b}}$ & $0.70 \pm 0.84$ & - & $0.80 \pm 0.71$ & $0.80 \pm 0.66$ \\
\hline
\end{tabular}

${ }^{\mathrm{a}} \mathrm{OHI}=$ Oral Hygiene Index; ${ }^{\mathrm{b}} \mathrm{CPITN}=$ Community Periodontal Index of Treatment Needs; ${ }^{\mathrm{p}} \mathrm{p}<0.05$ compared to baseline.

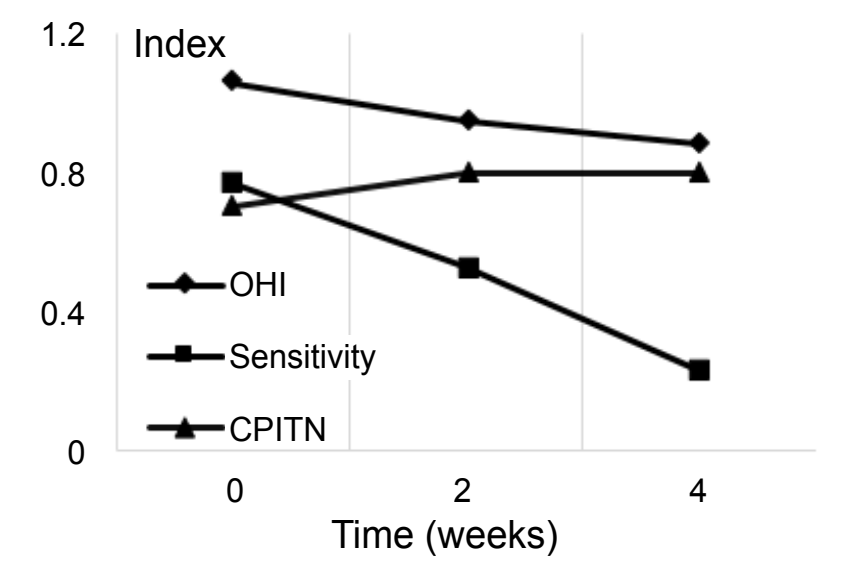

Figure 2: Comparison of indexes for SPLAT professional sensitive ultra toothpaste (Dentifrice A) contained $0.5 \%$ nHAP.

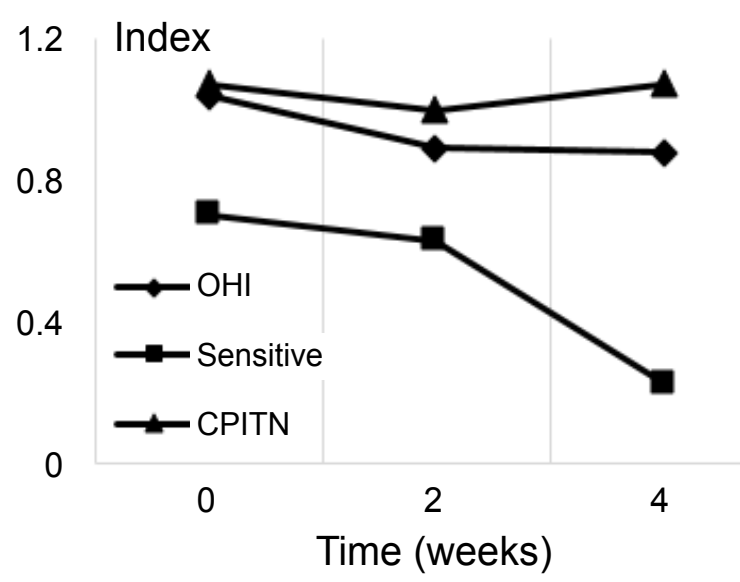

Figure 3: Comparison of indexes for Splat professional sensitive white toothpaste (Dentifrice B) containing $0.1 \%$ nHAP. 
Table 4: Difference in evaluated parameters between groups after 28 days of use (Mean \pm SD).

\begin{tabular}{|l|l|l|l|}
\hline & Group A & Group B & p-value \\
\hline $\mathrm{OHI}^{\mathrm{a}}$ & $-0.18 \pm 0.25$ & $-0.16 \pm 0.21$ & $\mathrm{p}>0.05$ \\
\hline Sensitivity & $-0.53 \pm 0.51$ & $-0.47 \pm 0.51$ & $\mathrm{p}>0.05$ \\
\hline Enamel mineralization & $-0.43 \pm 0.90$ & $-0.03 \pm 0.32$ & $\mathrm{P}=0.03$ \\
\hline CPITN $^{\mathrm{b}}$ & $0.10 \pm 0.40$ & $0.0 \pm 0.26$ & $\mathrm{p}>0.05$ \\
\hline
\end{tabular}

${ }^{\mathrm{a}} \mathrm{OHI}=$ Oral Hygiene Index; ${ }^{\mathrm{b}} \mathrm{CPITN}=$ Community Periodontal Index of Treatment Needs.

Table 3 shows the scores at each examination period for 60 patients for toothpaste Sensitive Ultra (Dentifrice B). Sensitivity scores declined steadily after the use of dentifrices was begun, decreasing by $67 \%$ in the end of the study period. After 4 weeks of treatment the $\mathrm{OHI}$ score shown a $15.4 \%$ reduction while other parameters have not changed significantly (Figure 3).

\section{Discussion}

The results of this exploratory study confirm those of previously published clinical studies that separately demonstrate the efficacy of toothpastes containing nanoHAP \% in the treatment of DH [13]. In this study, two $\mathrm{x} \%$ nHAP toothpastes achieved similar statistically significant reductions from baseline in $\mathrm{DH}$ over the 4-week treatment period. Both active treatments performed as expected with regard to improvements sensitivity scores, and in line with previously published clinical studies for these technologies [14].

Although this study was not formally powered to detect differences between treatments, use of both the active occluding toothpastes resulted in statistically significant improvements in tooth sensitivity compared with the baseline scores, with no statistically significant differences detected in gingival index, oral hygiene index, or remineralization (Table 4). The "natural desensitization process" may be related to a patient's oral hygiene practices. Plaque is a chemical stimulus which increases hypersensitivity if not removed. According to Chasens plaque removal through regular oral hygiene is an essential part of therapy for hypersensitivity [15]. However, in this study desensitization was not associated with plaque removal. It has been proposed that the nHAP deposits onto the dentine and occludes the tubules. In vitro studies have demonstrated occlusion of open dentine tubules following treatment with $\mathrm{x} \%$ nHAP. Evidence from in vitro and clinical studies confirms that nHAP occludes patent dentinal tubules, and supports the clinical efficacy of toothpastes containing $\mathrm{x} \% \mathrm{nHAP}$ for the effective relief of dentin hypersensitivity.

\section{Conclusions}

In conclusion, the results of this study support the short term efficacy of a $\mathrm{x} \%$ nHAP occlusion technology-based toothpaste for the relief of dentin hypersensitivity. Sensitivity relief was observed within 2 weeks of starting treatment and was continuing to improve at the end of the 4-week treatment period. An appropriately powered follow-up study is warranted for more extensive evaluation of the efficacy of the $x \%$ nHAP toothpaste formulation compared with other formulations.

\section{Conflicts of Interest}

All the authors received funding from SPLAT GLOBAL LLC at the time the study was conducted. The authors declare that there is no conflict of interest regarding the publication of this paper.

\section{Funding Statement}

Financial support for the conduct of the study and for editorial assistance in the preparation of the article was provided by SPLAT GLOBAL LLC.

\section{Author Contributions}

All the authors contributed to the design, conduct and reporting of the study. All authors had access to the final study report, made contributions to the development of the manuscript, had final responsibility for the decision to submit, and approved the submitted version.

\section{Clinical Relevance}

\section{Scientific rationale for study}

Dentin hypersensitivity is a very common phenomenon in patients. Knowing its prevalence and methods for accurate diagnosis is imperative to guide treatment decisions and develop new treatments. Although there are many therapeutic options for the treatment of dentin hypersensitivity, the management of this condition is still a problematic issue.

\section{Principal findings}

This clinical trial demonstrated a good therapeutic effect of nano-hydroxyapatite in the treatment of dentine hypersensitivity.

\section{Practical implications}

The use of oral care products with nano-hydroxyapatites, in particular SPLAT Professional Sensitive UItra toothpaste containing $0.5 \% \mathrm{nHAP}$, and SPLAT Professional Sensitive White toothpaste containing 0.1\% nHAP, reduce dentine hypersensitivity and have prolonged action. Such remedies should be recommended 
as part of comprehensive oral care, especially for dentin hypersensitivity patients.

\section{References}

1. Canadian Advisory Board on Dentin Hypersensitivity (2003) Consensus-based recommendations for the diagnosis and management of dentin hypersensitivity. J Can Dent Assoc 69: 221-226.

2. Clark D, Levin $L$ (2018) Tooth hypersensitivity treatment trends among dental professionals. Quintessence Int 49: 147-151.

3. Kopycka-Kedzierawski DT, Meyerowitz C, Litaker MS, Chonowski S, Heft MW, et al. (2017) Management of dentin hypersensitivity by national dental practice-based research network practitioners: Results from a questionnaire administered prior to initiation of a clinical study on this topic. BMC Oral Health 17: 41.

4. Porto IC, Andrade AK, Montes MA (2009) Diagnosis and treatment of dentinal hypersensitivity. J Oral Sci 51: 323332.

5. Brannstrom M (1963) Dentin sensitivity and aspiration of odontoblasts, J Am Dent Assoc 66: 366-370.

6. West NX (2008) Dentine hypersensitivity: Preventive and therapeutic approaches to treatment. Periodontol 2000 48: $31-41$.

7. West N, Seong J, Davies M (2014) Dentin hypersensitivity. Monogr Oral Sci 25: 108-122.

8. Holland GR, Narhi MN, Addy M, Gangarosa L, Orchardson R (1997) Guidelines for the design and conduct of clinical trials on dentine hypersensitivity. J Clin Periodontol 24: 808-813.

9. Hall C, Mason S, Cooke J (2017) Exploratory randomised controlled clinical study to evaluate the comparative efficacy of two occluding toothpastes-a $5 \%$ calcium sodium phosphosilicate toothpaste and an $8 \%$ arginine/calcium carbonate toothpaste-for the longer-term relief of dentine hypersensitivity. J Dent 60: 36-43.

10. Polson AM, Caton JG, Yeaple RN, Zander HA (1980) Histological determination of probe tip penetration into gingival sulcus of humans using an electronic pressure-sensitive probe. J Clin Periodontol 7: 479-488.

11. Schiff T, Dotson M, Cohen S, De Vizio W, McCool J, et al. (1994) Efficacy of a dentifrice containing potassium nitrate, soluble pyrophosphate, PVM/MA copolymer, and sodium fluoride on dentinal hypersensitivity: A twelve-week clinical study. J Clin Dent 87-92.

12. Bekes K, Heinzelmann K, Lettner S, Schaller HG (2017) Efficacy of desensitizing products containing $8 \%$ arginine and calcium carbonate for hypersensitivity relief in $\mathrm{MIH}$-affected molars: An 8-week clinical study. Clin Oral Investig 21: 2311-2317.

13. Gopinath NM, John J, Nagappan N, Prabhu S, Kumar ES (2015) Evaluation of dentifrice containing nano-hydroxyapatite for dentinal hypersensitivity: A randomized controlled trial. J Int Oral Health 7: 118-122.

14. Porcelli HB, Maeda FA, Silva BR, Miranda WG Jr, Cardoso PE (2015) Remineralizing agents: Effects on acid-softened enamel. Gen Dent 63: 73-76.

15. Chasens Al (1979) Periodontal disease, pathologic tooth migration and adult orthodontics. N Y J Dent 49: 40-43. 